\title{
Penrose Suggestion as to Pre Planck Era-Black Holes Showing Up in Present Universe Data Sets Discussed, with a Possible Candidate as to GW Radiation Which May Provide Initial CMBR Data
}

\author{
Andrew Walcott Beckwith \\ Physics Department, Chongqing University, Chongqing, China \\ Email: Rwill9955b@gmail.com, abeckwith@uh.edu
}

How to cite this paper: Beckwith, A.W. (2021) Penrose Suggestion as to Pre Planck Era-Black Holes Showing Up in Present Universe Data Sets Discussed, with a Possible Candidate as to GW Radiation Which May Provide Initial CMBR Data. Journal of High Energy Physics, Gravitation and Cosmology, 7, 1264-1277.

https://doi.org/10.4236/jhepgc.2021.74076

Received: June 24, 2021

Accepted: August 27, 2021

Published: August 30, 2021

Copyright (๑) 2021 by author(s) and Scientific Research Publishing Inc. This work is licensed under the Creative Commons Attribution International License (CC BY 4.0).

http://creativecommons.org/licenses/by/4.0/

\begin{abstract}
What we are doing is three-fold. First, we examine the gist of the Penrose suggestion as to signals from a prior universe showing up in the CMBR. i.e., this shows up as data in the CMBR. Second, we give a suggestion as to how super massive black holes could be broken up s of a prior Universe cycle by pre big bang conditions, with say millions of pre-Planck black holes coming up out of a breakup of prior universe black holes. Three, we utilize a discussion as to Bose Einstein Condensates set as Gravitons as to composing the early universe black holes. The BEC formulation gives a number $\mathrm{N}$ of gravitons, linked to entropy, per black hole, which could lead to contributions to the alleged CMBR perturbations, which were identified by Penrose et al.
\end{abstract}

\section{Keywords}

Minimum Scale Factor, Cosmological Constant, Space-Time Bubble, Penrose Singularity, Prior Universe Black Holes

\section{First What is the Penrose Suggestion as to CMBR Data Set and pre Universe Massive Black Holes?}

The abstract has a clue, as part of [1] states as to what we want to explain in the CMBR, i.e. circular rings in the CMBR "data".

In [1] there is a well crafted suggestion as to V. G. Gurzadyan, R. Penrose as to an initial quote. 


\section{Quote}

The significance of individual low-variance circles in the true data has been disputed; yet a recent independent analysis has confirmed CCC's expectation that $\mathrm{CMB}$ circles have a non-Gaussian temperature distribution. Here we examine concentric sets of low-variance circular rings in the WMAP data, finding a highly non-isotropic distribution.

\section{End of quote}

Here are the nuts and bolts as to what Penrose cosmology is about i.e. [2].

1) There is initial inflationary expansion of the Universe, but the caveat is that matter-energy is sucked up in super massive black holes. i.e., rather than have a purported infinite expansion, and we see the following dynamic.

We connect a countable sequence of open Friedmann-Lemaitre-RobertsonWalker metric (FLRW) spacetimes, each representing a Big Bang followed by an infinite future expansion. Penrose noticed that the past conformal boundary of one copy of FLRW spacetime can be "attached" to the future conformal boundary of another, after an appropriate conformal rescaling. Result $g_{a b}$ reset in a conformal reset with matter from black holes collected and reset to a new value of $g_{a b}$ at the start of cosmological expansion with matter-energy from black holes being recycled conformally to a new expansion cycle [2].

2) Next, let us view the Penrose suggestion as to Black holes from a prior universe.

In order to see this, consider a suggestion as to black holes, being the template for a start to the present universe given by [3], and also [4] which has the Penrose suggestion of an imprint of a prior Universe black holes having an effect upon the CMBR spectrum. The CMBR spectrum is a real datum, but the worth of getting this information would be in terms of having what was said in [4] as to the "ghost" of prior universe black hole radiation. To get a glimpse of where this is going the author invites readers to look at [5] as to the cosmic maelstrom such "signals" would have to pass through.

Quote from page 2 of [4] namely.

Figure 1: A conformal diagram representing the effect of a highly energetic event occurring at the space-time point $\mathrm{H}$. In CCC, $\mathrm{H}$ is taken to be a Hawking point, where virtually the entire Hawking radiation of a previous-aeon supermassive black hole is concentrated at $\mathrm{H}$ by the conformal compression of the hole's radiating future. The horizontal line at the bottom stands for the crossover surface dividing the previous cosmic aeon from our own and describes our conformally stretched Big Bang. In conventional inflationary cosmology, X would represent the graceful exit turn-off of inflation. In each case, the future light cone of $\mathrm{H}$ represents the outer causal boundary of physical effects initiated at $\mathrm{H}$, and such effects can reach $\mathrm{D}$ only within the roughly 0.08 radian spread indicated at the top of the diagram.

\section{End of quote}




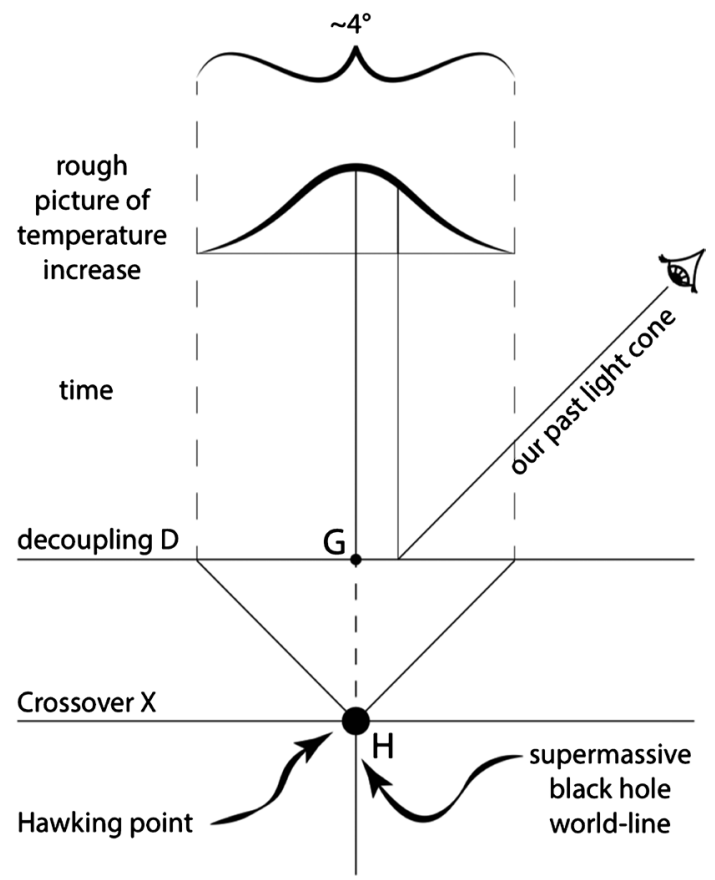

Figure 1. As given in [4] which has competing black hole radiation, and can we see this today in the CMBR?

\section{What Can We Expect from the Transition from a Prior Universe to the Planckian Regime of Micro Black Holes? A Transition from Initially Gigantic Black Holes to Micro Black Holes}

1) In a word, we would likely have in the prior universe MASSIVE black hole, which would be broken up into millions (billions?) of Planck sized.

In a word the GW radiation and thermal/photonic input would have to fight through a thicket of pairs of micro black holes which would be in binary configuration generating their own GW background.

We first will discuss this "binary black holes" signal background which the Planckian early Universe stars would have to impinge upon, in order to come to our attention.

Now for the discussion of the millions (more than that) of micro sized black hole pairs which would create a generalized GW signature.

To evaluate the above in terms of our model, we need to refer to a formula given in [5], on page 16 of that document which reads as a change in power from rotating Planck sized black holes separated say by a Planck length.

$$
\begin{aligned}
& \dot{E}=\mathrm{GW} \text { change in energy }=\frac{32\left(M_{1} M_{2}\right)^{2}\left(M_{1}+M_{2}\right)}{5 \cdot R^{2} M_{\text {Planck }}^{5}} \\
& \underset{M_{1}=M_{2}=M_{\text {Planck }}}{\longrightarrow} \frac{64}{5 \cdot R^{2}(\text { Planck length })} \\
& \equiv \text { Change in power from Rotating binary black holes }
\end{aligned}
$$

For $M$ about the size of a Planck sized black hole, it likely would fade out al- 
most too quickly to be very measurable.

2) We also can consider the following Gravitons as a Bose-Einstein condensate in low mass Black holes, and its relevance to signal propagation.

This is a way to get measurable GW signals from a black hole, which have a chance of being detected.

We will be looking at [6] specifically page 181, where we have the following scaling arguments to work through, if Gravitons are BEC for small Black holes. The following are scaling value to consider, if we want BEC.

Why we consider BECs and Equation (2), i.e., if there is a break up of massive black holes into say Planck mass sized black holes, as or about the Planck era, very likely will not have a surviving signal which has a chance of being measurable in the CMBR data. i.e., the discussion of Equation (2) below uses the device of having BEC condensation in gravitons for masses up to about 10 grams or so, and in doing so a dodge as to getting entropy counts per black hole.

That is after the black hole masses, as given in Equation (2) are likely built up by the consolidation of two mini black holes going through an inspiral collapse, as has been modeled in GW.

$$
\begin{aligned}
& m \approx \frac{M_{P}}{\sqrt{N_{\text {gravitons }}}} \cdot M_{P} \\
& M_{B H} \approx \sqrt{N_{\text {gravitons }}} \cdot l_{P} \\
& R_{B H} \approx \sqrt{N_{\text {gravitons }}} \cdot l_{\text {gravitons }} \\
& S_{B H} \approx k_{B} \cdot N_{\text {gran }}
\end{aligned}
$$

Here, the first term, $m$, is in the effective mass of a graviton. This is my take as to how to make all this commensurate as to special relativity.

$$
\begin{gathered}
m \approx \frac{m_{g}}{\sqrt{1-\left(\frac{v_{g}}{c}\right)^{2}}} \approx \frac{M_{P}}{\sqrt{N_{\text {gravitons }}}} \approx 10^{-10} \text { grams } \\
\therefore N_{\text {gravitons }} \approx 10^{10}
\end{gathered}
$$

With this, if say one has a 1 gram black hole, about $10^{5}$ times larger than a Planck mass, one would be having say an entropy generated this way of about $10^{10}$, assuming Planck normalization.

\section{So, Then What are the Number of Gravitons Emitted via a Spinning Planck Sized Black Holes Component Binary in Terms of Gravitons? [7] What does This Say about an Optimal BH Size as to Perhaps See Measurable GW/Graviton Generation Effects}

Likely from the situation in [7] for items as of about a Planck length, and involving Planck sized masses, we would see the following equation for a rotating 
rod, of mass $M$, and of velocity $V$, of its end, for Graviton production.

$$
\begin{aligned}
& N_{g} \approx \frac{32}{45} \cdot \frac{G}{\hbar c} \cdot M^{2}(\text { rod }) \cdot\left(\frac{V_{\text {rod tip }}}{c}\right)^{4} \propto 7.5 \times 10^{-8} \cdot(M(\text { rod }))^{2} \\
& \Rightarrow N_{g} \geq 1 \text { iff } M(\text { rod }) \geq 4 \times 10^{6} \text { grams }
\end{aligned}
$$

If we have an equivalent situation w.r.t. 2 black holes in a binary state, we would likely need to have approximately black holes of masses $10^{5} \mathrm{~g}$ to $10^{6}$ grams, i.e., $10^{10}$ to $10^{11}$ times larger than Planck mass, in order to have a measurable GW/Graviton signal which would be commensurate with experimental data sets. If we had say $10^{5}$ to $10^{6} \mathrm{~g}$ black holes, then the value of gravitons released per second, from a BEC condensate of Gravitons for a mini black hole would me many times larger than Equation (4) above.

We don't know the exact values, but this leads to our next point, which is the stages of Black holes, before the Planckian era, to at the point of time (and space) where 1 to $10^{5}$ gram black holes would be composed of Gravitons by BEC condensation of Gravitons, for a release in.

1) Considering this, what can we say about the regimes of $\mathrm{BH}$ masses, just before the Planckian era, during the Planckian era, and right after the Planckian era?

We are assuming the following. A moderately large $\left(10^{6}\right)$ or more number of super massive black holes which would be in the center of galaxies, and which would be broken up and recycled in the CCC cosmology regime, with masses dropping from about $10^{41}$ grams, down to about $10^{-4}$ to $10^{-5}$ grams, before recombination by Planck era recombination into a tier of black holes which would be at least 1 gram in mass, scaling up to $10^{5}$ grams in mass so as to allow for BEC generation of gravitons through entropy production as in Equations (2) and (3) above.

In doing so, we purport to use the datum given in [7] that masses of say much lower than $10^{5}$ to $10^{6}$ grams for black holes likely do not have much chance of producing gravitons which would be detectable in the present era. Indeed, a minimum mass of about 1 to 10 grams for a black hole would be needed for a Bose Einstein condensation via gravitons for a "light-low mass" black hole which would be able to by Equations ((2), (4) and (5)) to have at least $10^{10}$ gravitons per second generated (entropy for a BEC black hole).

2) We then would to a round off approximation state this hierarchy of $\mathrm{BH}$ behavior and size to consider Table 1 .

\section{Why We Would have the Figures From III.B to Consider For Contributions to the CMBR And The Penrose Suggestion the Formula Which Is For Luminosity from a Black Hole and in Page 16 Of Reference [5] the Text States That the Two Black Holes Emit GW with a Wave Frequency 2 Times The Rotation Frequency of the Orbit of the Two Black Holes to Each Other}

If we assume that we are still using this approximation above, from [5] we can 
Table 1. Scaling of Mass of Black holes, and their purported number, if CCC cosmology (Penrose) assumed for GW radiation release (may affect the CMBR).

\begin{tabular}{|c|c|c|}
\hline End of Prior Universe time frame & $\begin{array}{c}\text { Mass(black hole): } \\
\text { super massive end of } \\
\text { time } \mathrm{BH} \\
1.98910^{+41} \text { to about } \\
10^{44} \text { grams }\end{array}$ & $\begin{array}{c}\text { Number(black holes) } \\
10^{6} \text { to } 10^{9} \text { of them usually from center } \\
\text { of galaxies }\end{array}$ \\
\hline & Mass(black hole) & Number(black holes) \\
\hline $\begin{array}{c}\text { Planck era Black hole formation } \\
\text { Assuming start of merging of micro } \\
\text { black hole pairs }\end{array}$ & $\begin{array}{c}10^{-5} \text { to } 10^{-4} \text { grams (an } \\
\text { order of magnitude of } \\
\text { the Planck mass } \\
\text { value) }\end{array}$ & $\begin{array}{l}10^{40} \text { to about } 10^{45} \text {, assuming that there } \\
\text { was not too much destruction of } \\
\text { matter-energy from the Pre Planck } \\
\text { conditions to Planck conditions }\end{array}$ \\
\hline $\begin{array}{l}\text { Post Planck era black holes with the } \\
\text { possibility of using Equation (2) to } \\
\text { have say } 10^{10} \text { gravitons/second } \\
\text { released per black hole }\end{array}$ & $\begin{array}{l}\text { Mass(black hole) } \\
10 \text { grams to say } 10^{6} \\
\text { grams per black hole }\end{array}$ & $\begin{array}{l}\text { Number(black holes) } \\
\text { Due to repeated Black hole pair } \\
\text { forming a single black hole multiple } \\
\text { times } \\
10^{20} \text { to at most } 10^{25}\end{array}$ \\
\hline
\end{tabular}

see support for our choice of Planck length as the minimum separation distance between the two black holes via using Plank units normalized to 1 as yielding.

$$
\begin{aligned}
& R(\text { separation }) \simeq r_{g}^{\text {eff }}=\frac{\left(M_{1}+M_{2}\right)}{\left(M_{\text {Planck }}\right)^{2}} \\
& \left.\underset{M_{1}=M_{2}=M_{\text {Planck }} \underset{M_{\text {Planck }}=1}{\longrightarrow}}{\longrightarrow} 1 \equiv R \text { (Planck length }\right)
\end{aligned}
$$

Going to Clifford Will, [8] we see on page 252 a loss or shrinkage of the period for the rotating black hole pair defined by $\mathrm{P}$.

$$
\dot{P} / P=\frac{\mathrm{d} P}{\mathrm{~d} t} \cdot \frac{1}{P}=-\frac{3}{2} \frac{\dot{E}}{E}
$$

Whereas, with the Mechanics version of $P$ for a sphere to be defined by, where $M$ is a mass of a star, and we assume a binary system with two masses of equal mass $M$, so that, if $R$ is the separation between the two masses [9] on page 188 would be

$$
\begin{aligned}
& P=R \sqrt{\frac{2 \pi^{2} R}{G \cdot M}} \underset{M_{1}=M_{2}=M_{\text {Planck }} \overline{M_{\text {Planck }}=1}}{\longrightarrow} \\
& R \text { (Planck length }) \sqrt{\frac{2 \pi^{2} R(\text { Planck length })}{G \cdot M(\text { Planck })}}
\end{aligned}
$$

For Planck sized masses, this means that the period of the binary plank mass black hole pair would be vanishingly small, the frequency of rotation would be half that of the GW emitted by these two Planck mass black holes which would collapse into each other. Note that the frequency we have stated for this last step, is given in Equation (9). i.e., could we have the following quantization contribution to initial frequency?

Our final concluding point to this chapter is to review the physics of Figure 1, and then to ask, can we ascertain the GW radiation of Planck era black hole stars in a binary configuration contributing to a buildup of generating frequency get- 
ting. If

$$
\Delta E \Delta t \approx \hbar \equiv \hbar \omega \Delta t \approx \hbar \omega \cdot\left(\frac{2}{3 a_{\min }}\right)^{1 / \gamma} \Rightarrow \omega \approx \hbar^{-1} \cdot\left(\frac{2}{3 a_{\min }}\right)^{-1 / \gamma}
$$

We claim that if we take the energy as consistent with a change in value as given by Equation (1) that this will lead to a frequency which may, if $a_{\text {min }} \approx 10^{-25}-10^{-20}$ (range from $10^{-25}$ to $10^{-20}$ ) lead to

$$
\omega \approx \hbar^{-1} \cdot\left(\frac{3}{2}\right)^{1 / \gamma} \cdot 10^{25 / \gamma} \propto\left(\frac{3}{2}\right)^{1 / \gamma} \cdot 10^{25 / \gamma} \mathrm{Hz}
$$

Whereas note that the frequency is, say dependent upon the choice of $\gamma$ and that this could be very different from the Planck frequency given below

$$
\omega_{P} \approx 1.885 \times 10^{43} \mathrm{~Hz}
$$

We have then that if one had a redshift, of $z \sim 10^{25}$, that this would mean a present value of frequency as of about $1 \mathrm{~Hz}$, whereas we can consider what would be gained by looking at the contribution near the CMBR, i.e., $z \sim 1100$ or so for the CMBR, whereas this would mean roughly that we would be looking in the regime of the CMBR.

$$
\omega_{\text {signal from Planck to CMBR }} \propto\left(\frac{3}{2}\right)^{1 / \gamma} \cdot 10^{25 / \gamma} \times 10^{-3} \mathrm{~Hz}
$$

However, we have in doing this, that the duration of this frequency signal would be very minimal, i.e., due to the decay of the period, this would be going on for less than a nanosecond.

If so then we would need to refer to Equation (2) and the value of

$$
\begin{aligned}
& E_{\mathrm{BEC} \text { Graviton }} \approx \frac{k_{\mathrm{B}} T_{\mathrm{BH}}}{2} \approx \frac{k_{\mathrm{B}} \times 10^{-5} \times T_{P}}{2} \\
& \Rightarrow \omega_{\mathrm{BEC} \text { Graviton }} \propto 10^{-5} \times 10^{43} \mathrm{~Hz} \approx 10^{38} \mathrm{~Hz} \\
& \Rightarrow \omega_{\mathrm{BEC} \text { Graviton to CMBR }} \approx 10^{38} \times 10^{-3} \mathrm{~Hz}
\end{aligned}
$$

Needless to state, that unlike the case of (12), one would likely have the duration of the signal last long enough as to imprint directly on the CMBR i.e., look at [9] and also For this I refer to the Zeldovich 4 conference Abhay Ashtekar presentation [10].

Ashtekar referred to a removal of bogus data points in the CMBR, as to the Figure 2 below, i.e., Figure 2.

Now looking at what was discussed by Abhay Ashtekar in Zeldovich 4. On September 7, 2020 [10].

In our Figure 1, we copy what was done by Ashtekar, in Zelsovich 4 as to what was part of anisotropic fits to the $\mathrm{E}$ and $\mathrm{B}$ polarization, as given is made easier, if there is a non singular start to the universe which I discussed in detail in [11], and that further polarization states which may be analyzed in detail could be ascertained in [12].

If one has a nonsingular start to the universe, modeled on a muti universe 


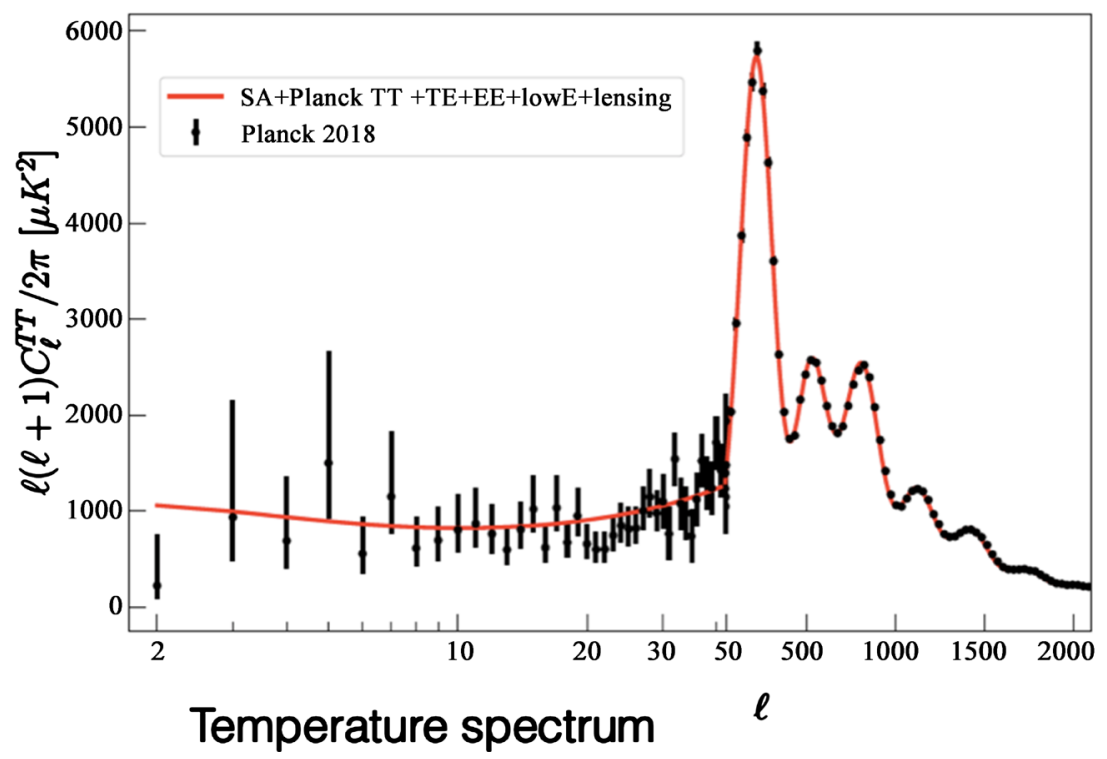

Figure 2. Corrected CMBR data points included in this plot.

generalization of Penrose CCC cosmology as is argued in [11] then the details of a break up of black holes would not be so startling, i.e., these are the details from [11] as given by the following generalization of CCC cosmology, and this is from [11].

\section{Looking Now at the Modification of the Penrose CCC (Cosmology)}

We now outline the generalization for Penrose CCC (Cosmology) just before inflation which we state we are extending Penrose's suggestion of cyclic universes, black hole evaporation, and the embedding structure our universe is contained within. This multiverse has BHs and may resolve what appears to be an impossible dichotomy. The text following is largely from [11] and has serious relevance to the final part of the conclusion. That there are $N$ universes undergoing Penrose 'infinite expansion' (Penrose) [2] contained in a mega universe structure with Hawking radiation from decaying black holes. If each of the $N$ universes is defined by a partition function, called $\left\{\Xi_{i}\right\}_{i=N}^{i=1}$, then there exist an information ensemble of mixed minimum information correlated about $10^{7}-10^{8}$ bits of information per partition function in the set $\left.\left\{\Xi_{i}\right\}_{i=N}^{i=1}\right|_{\text {before }}$, so minimum information is conserved between a set of partition functions per universe [13].

$$
\left.\left.\left\{\Xi_{i}\right\}_{i=N}^{i=1}\right|_{\text {before }} \equiv\left\{\Xi_{i}\right\}_{i=N}^{i=1}\right|_{\text {after }}
$$

However, there is non-uniqueness of information put into partition function $\left\{\Xi_{i}\right\}_{i \equiv N}^{i \equiv 1}$. Also Hawking radiation from black holes is collated via a strange attractor collection in the mega universe structure to form a new inflationary regime for each of the $N$ universes represented.

Our idea is to use what is known as CCC cosmology [13], which can be thought of as the following. First. Have a big bang (initial expansion) for the universe which is represented by $\left\{\Xi_{i}\right\}_{i=N}^{i=1}$. Verification of this mega structure 
compression and expansion of information with stated non-uniqueness of information placed in each of the $N$ universes favors ergodic mixing of initial values for each of $N$ universes expanding from a singularity beginning. The $n_{f}$ stated value, will be using ( $\mathrm{Ng}, 2008) S_{\text {entropy }} \sim n_{f}$. [13] [14]. How to tie in this energy expression, as in Equation (12) will be to look at the formation of a nontrivial gravitational measure as a new big bang for each of the $N$ universes as by $n\left(E_{i}\right)$. the density of states at energy $E_{i}$ for partition function [13] [15].

$$
\left\{\Xi_{i}\right\}_{i=1}^{i \equiv N} \propto\left\{\int_{0}^{\infty} \mathrm{d} E_{i} \cdot n\left(E_{i}\right) \cdot \mathrm{e}^{-E_{i}}\right\}_{i=1}^{i \equiv N}
$$

Each of $E_{i}$ identified with Equation (9) above, are with the iteration for $N$ universes [13], and (Penrose, 2006) [2]. Then the following holds, by asserting the following claim to the universe, as a mixed state, with black holes playing a major part, i.e.

\section{CLAIM 1}

See the below [13] representation of mixing for assorted $N$ partition function per CCC cycle

$$
\left.\left.\frac{1}{N} \cdot \sum_{j=1}^{N} \Xi_{j}\right|_{j \text { before nucleation regime }} \underset{\text { vacuum nucleation tranfer }}{\longrightarrow} \Xi_{i}\right|_{i \text { fixed after nucleation regime }}
$$

For $N$ number of universes, with each $\left.\Xi_{j}\right|_{j \text { before nucleation regime }}$ for $j=1$ to $N$ being the partition function of each universe just before the blend into the RHS of Equation (16) above for our present universe. Also, each independent universes as given by $\left.\Xi_{j}\right|_{j \text { before nucleation regime }}$ is constructed by the absorption of one to ten million black holes taking in energy i.e., (Penrose) [2]. Furthermore, the main point is done in [11] in terms of general ergodic mixing [13].

Claim 2

$$
\left.\left.\Xi_{j}\right|_{j \text { before nucleation regime }} \approx \sum_{k=1}^{\text {Max }} \tilde{\Xi}_{k}\right|_{\text {black holes jth universe }}
$$

What is done in Claim 1 and 2 [11] is to come up as to how a multi dimensional representation of black hole physics enables continual mixing of spacetime largely as a way to avoid the Anthropic principle [11], as to a preferred set of initial conditions.

\section{Conclusion, i.e. If One Has A Nonsingular Start of Expansion of the Universe and Ergodic Mixing of Initial Conditions of Space-Time From Other Universes, How does This Relate to the Breaking up of Bhs from Table 1?}

In [11] in order to do away with the Anthropic principle, the following references in terms of Ergodic mixing of the Partition function of the Universe was utilized, as far as a multiverse. But there is one final piece. Assume that we have

$$
\omega_{\text {Earth orbit }} \leq 10^{-25} \omega_{\text {initial era }}
$$

We will be of course assuming an equivalence [16] between a graviton count 
and information, as given by [17], and we can in future work compare this with the Rosen [19] value of energy for a mini universe of (from a Schrodinger equation) with ground state mass of $m=\sqrt{\pi} M_{\text {Planck }}$ and an energy of

$$
E_{\hat{n}}=\frac{-G m^{5}}{2 \pi^{2} \hbar^{2} \hat{n}^{2}}
$$

Our preliminary supposition is that Equation (19) could represent the initial energy of a Pre Planckian Universe and that Equation (20) would be thermally based energy dumped into the space-time bubble assumed to be in [11], i.e.

$$
E(\text { universe })=10^{41} \times E_{\text {BEC Graviton }} \approx 10^{41} \times\left(\frac{k_{B} T_{B H}}{2} \approx \frac{k_{B} \times 10^{-5} \times T_{P}}{2}\right)
$$

be the thermal energy dumped in due to the use of Cyclic Conformal cosmology. Here we specify that initially it would have that the value of Equation (20) would exactly counter balance the energy given in a negative form by Rosen as of Equation (19).

Now use the following approximation of the Universe, initially having the entropy of a black hole, i.e., we are using $\mathrm{Ng}$ Infinite Quantum statistics, [20], while Area denotes the surface area of the regime of space-time.

$$
S_{\text {Universe }} \propto S_{B H} \cong \frac{A(\text { area })}{4 \cdot \ell_{\text {Planck }}^{2}} \approx \frac{9 n_{Q}}{4} \approx n_{\text {graviton }}
$$

This way of noting entropy and the signals of the prior Universe Black holes being generated secondarily is a surface area which is commensurate with the utilization of Equation (2) for BEC condensation by Gravitons for Early Universe black holes. This is in tandem with the quantum fluctuations as seen in Figure 3 below. Also see Appendix A below, as well as the physics, of [4] [18] [19] and [20]

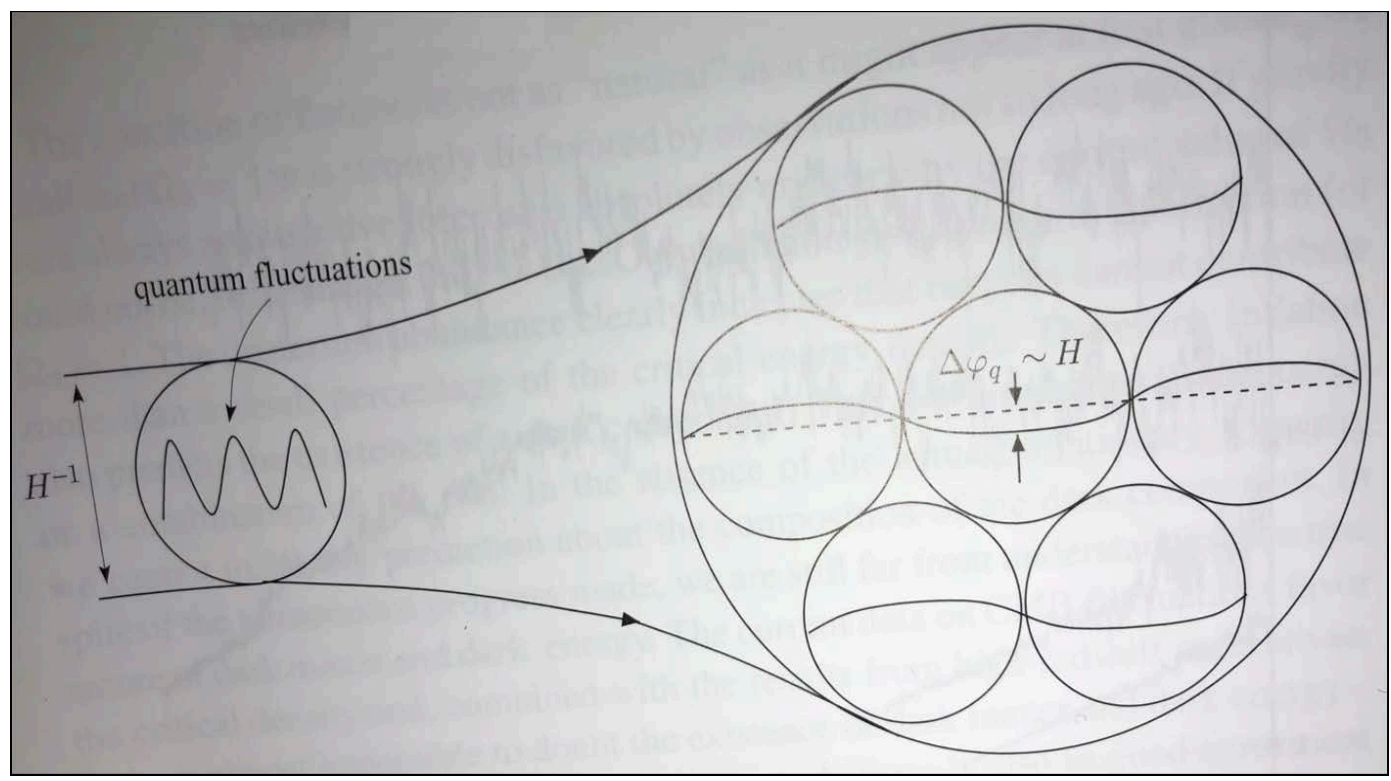

Figure 3. Which is from reference [11] [15]. 
The bubble nucleation, plus the details of cosmology as presented in [21] leading to

Quote

For thirty years Oxford Mathematician Roger Penrose has challenged one of the key planks of Cosmology, namely the concept of Inflation, now over 40 years old, according to which our universe expanded at an enormous rate immediately after the Big Bang. Instead, fifteen years ago, Penrose proposed a counter-concept of Conformal Cyclic Cosmology by which Inflation is moved to before the Big Bang and which introduces the idea of preceding aeons. The concept has been disputed by most physicists, but Roger and colleagues believe that new evidence has come to light which requires closer inspection and argument-the research is published today in the Monthly Notices of the Royal Astronomical Society (MNRAS).

Recent analysis of the Cosmic Microwave Background (CMB) by Roger, Daniel An, Krzysztof Meissner and Pawel Nurowski has revealed, both in the Planck and WMAP satellite data (at $99.98 \%$ confidence), a powerful signal that had never been noticed previously, namely numerous circular spots $\sim 8$ times the diameter of the full moon. The brightest six (Figure 1) are $\sim 30$ times the average $\mathrm{CMB}$ temperature variations seen at precisely the same locations in the Planck and WMAP data. These spots were overlooked previously owing to a belief that the very early exponentially expanding inflationary phase of standard cosmology should have obliterated any such features Figure 4.

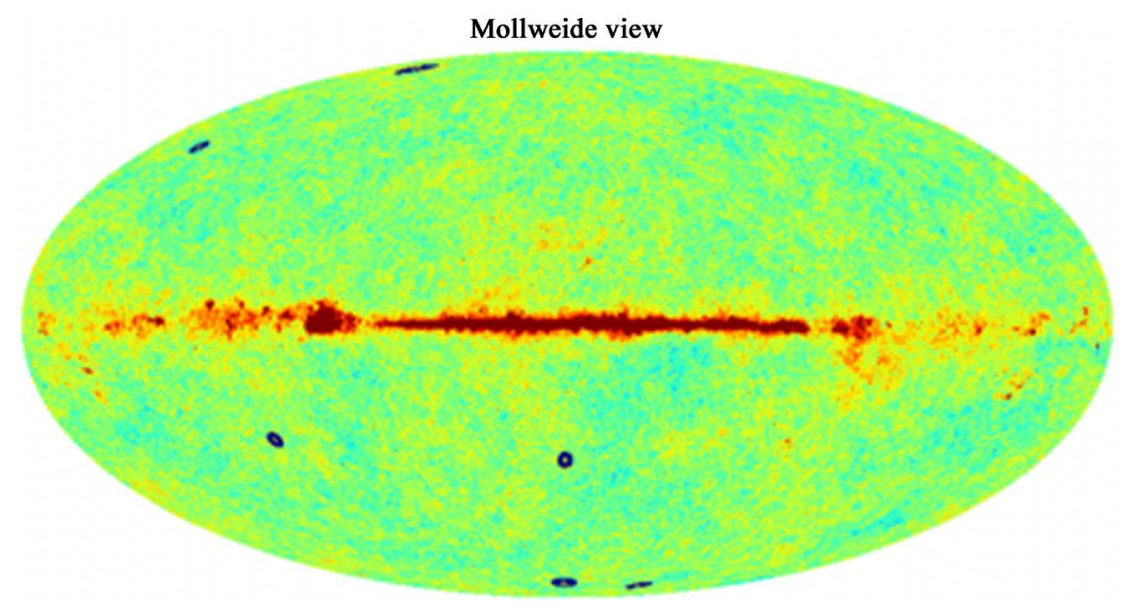

Figure 4. Penrose addendum to CMBR including pre Big bang contributions.

End of quote

We submit that judicious application of Equation (2) plus Table 1 above will lead to this phenomenon.

\section{Conflicts of Interest}

The author declares no conflicts of interest regarding the publication of this paper. 


\section{References}

[1] Gurzadyan, V.G. and Penrose, R. (2013) On CCC-Predicted Concentric Low-Variance Circles in the CMB Sky. The European Physical Journal Plus, 128, 22.

https://arxiv.org/abs/1302.5162 https://doi.org/10.1140/epjp/i2013-13022-4

[2] Penrose, R. (2006) Before the Big Bang: An Outrageous New Perspective and Its Implications for Particle Physics. Proceedings of the EPAC 2006, Edinburgh, 26-30 June 2006, 2759-2762.

[3] Popławski, N.J. (2019) Black Hole Genesis and Origin of Cosmic Acceleration. https://arxiv.org/abs/1912.02173

[4] An, D., Meissner, K.A., Nurowski, P. and Penrose, R. (2018) Apparent Evidence for Hawking Points in the CMB Sky. https://arxiv.org/abs/1808.01740

[5] Dolgov, A.D. and Ejlli, D. (2011) Relic Gravitational Waves from Light Primordial Black Holes. Physical Review D, 84, Article ID: 024028.

https://arxiv.org/abs/1105.2303

https://doi.org/10.1103/PhysRevD.84.024028

[6] Chavanis, P.H. (2012) Self Gravitating Bose-Einstein Condensates. In: Calmet, X., Ed., Quantum Aspects of Black Holes, Fundamental Theories of Physics, Vol. 178, Springer Nature, Cham, 151-194. https://doi.org/10.1007/978-3-319-10852-0_6

[7] Murphy, G.L. (1978) Gravitons from a Spinning Rod. Australian Journal of Physics, 31, 205-207. https://www.publish.csiro.au/ph/pdf/PH780205 https://doi.org/10.1071/PH780205

[8] Will, C.M. (2018) Theory and Experiment in Gravitational Wave Physics. 2nd Edition, Cambridge University Press, New York.

[9] Passaglia, S., Hu, W. and Motohashi, H. (2019) Primordial Black Holes and Local Non-Gaussianity in Canonical Inflation. Physical Review D, 99, Article ID: 043536. https://arxiv.org/abs/1812.08243

[10] Abhay Ashtekar. http://www.icranet.org/images/stories/Meetings/ZM4/presentations/Ashtekar.pdf

[11] Beckwith, A. (2021) A Solution of the Cosmological Constant, Using Multiverse Version of Penrose CCC Cosmology, and Enhanced Quantization Compared. Journal of High Energy Physics, Gravitation and Cosmology, 7, 559-571. https://doi.org/10.4236/jhepgc.2021.72032

[12] Poplawski, N. (2011) Cosmological Constant from QCD Vacuum and Torsion. Annals of Physics (Berlin), 523, 291. http://arxiv.org/abs/1005.0893v1 https://doi.org/10.1002/andp.201000162

[13] Dye, H. (1964) On the Ergodic Mixing Theorem. http://www.ams.org/journals/tran/1965-118-00/S0002-9947-1965-0174705-8/S00029947-1965-0174705-8.pdf

[14] Naselsky, P.D., Novikov, D. and Novikov, I. (2006) The Physics of the Cosmic Microwave Background. Cambridge University Press, Cambridge. https://doi.org/10.1017/CBO9780511536373

[15] Mukhanov, V. (2005) Physical Foundations of Cosmology. Cambridge University Press, New York. https://doi.org/10.1017/CBO9780511790553

[16] Rosen, N. (1993) Quantum Mechanics of a Miniuniverse. International Journal of Theoretical Physics, 32, 1435-1440. https://doi.org/10.1007/BF00675204

[17] Haranas, I. and Gkigkitzis, I. (2014) The Mass of Graviton and Its Relation to the Number of Information According to the Holographic Principle. International Scho- 
larly Research Notices, 2014, Article ID: 718251.

https://doi.org/10.1155/2014/718251

[18] Ng, Y.J. (2007) Holographic Foam, Dark Energy and Infinite Statistics. Physics Letters B, 657, 10-14. https://arxiv.org/abs/gr-qc/0703096

https://doi.org/10.1016/j.physletb.2007.09.052

[19] Jow, D.L. and Scott, D. (2020) Re-evaluating evidence for Hawking points in the CMB. Journal of Cosmology and Astroparticle Physics, 2020, arXiv: 1909.09672. https://doi.org/10.1088/1475-7516/2020/03/021

[20] Letzter, R. (2018) Physicists Think They've Spotted the Ghosts of Black Holes from Another Universe.

https://www.livescience.com/63392-black-holes-from-past-universes.html

[21] Hawking Points in the Cosmic Microwave Background-A Challenge to the Concept of Inflation. https://www.maths.ox.ac.uk/node/36137 


\section{Appendix A. Examining How Many Gravitons Might be Produced by Initially Planck sized Black Holes}

From [5] Alexander D. Dolgov, Damian Ejlli inform us that a mass of a primordial black hole is

$$
M(\text { early black hole }) \approx 4 \times 10^{38} \cdot\left(\frac{t(\text { formation })}{\text { Sec }}\right) \cdot g(\text { grams })
$$

A Planck mass is of the value $10^{-5}$ grams, i.e. almost, is then obtainable when

$$
\begin{aligned}
& t(\text { formation }) \approx 10^{-43} \text { Sec } \\
& \left.\Rightarrow M(\text { early black hole }) \approx 10^{-5} g \text { (grams }\right)
\end{aligned}
$$

Note that $t$ (formation $) \approx 10^{-43} \mathrm{Sec} \geq 5.39 \times 10^{-44} \mathrm{~s}=$ Planck time leads to almost a Planck Mass

$$
2.176434(24) \times 10^{-5} \mathrm{~g}=M(\text { Planck mass })
$$

The mechanism of how Plank sized black holes could generate GW comes from, as given in [5] initial friction in the early universe environment, leading to coupling of early primordial binary black hole systems which in turn would collapse and form larger black holes, i.e., in fact the argument in [20] is stated in page 15 as follows.

Quote

For PBH masses below a few grams dynamical friction would be an efficient mechanism of $\mathrm{PBH}$ cooling leading to frequent binary formation. Moreover, dynamical friction could result in the collapse of small PBHs into much larger $\mathrm{BH}$ with the mass of the order of $\mathrm{Mb}$ (18). This process would be accompanied by a burst of GW emission.

End of quote

What is called $\mathrm{Mb}$ in this situation is given by, in [5] on page 4

Quote

As we see in what follows, generation of gravitational waves would be especially efficient from such high density clusters of primordial black holes. Let us assume that the spectrum of perturbations is the flat Harrison-Zeldovich one and that a perturbation with some wavelength $\lambda$ crossed horizon at moment $t$ in. The mass inside horizon at this moment was.

$\mathrm{Mb}(\mathrm{t}$ in $)=\mathrm{m} 2 \mathrm{P}$ lt in. (4)

It is the mass of the would-be high density cluster of PBHs

End of quote

We then from here have the mechanism of black hole formation comes from binary pair formation of small black holes which collapse into a larger set of black holes. This chain of BH pair production and collapse would then lead to an accretion procedure along the lines of Equation (21). Eventually these black hole clusters would form the mega black holes as seen in the center of spiral galaxies. 